\title{
The hidden geometric character of relativistic quantum mechanics
}

\author{
José B. Almeida \\ Universidade do Minho, Physics Department \\ Braga, Portugal, email: bda@fisica.uminho.pt
}

\begin{abstract}
Geometry can be an unsuspected source of equations with physical relevance, as everybody is aware since Einstein formulated the general theory of relativity. However efforts to extend a similar type of reasoning to other areas of physics, namely electrodynamics, quantum mechanics and particle physics, usually had very limited success; particularly in quantum mechanics the standard formalism is such that any possible relation to geometry is impossible to detect; other authors have previously trod the geometric path to quantum mechanics, some of that work being referred to in the tex. In this presentation we will follow an alternate route to show that quantum mechanics has indeed a strong geometric character.
\end{abstract}

The paper makes use of geometric algebra, also known as Clifford algebra, in 5-dimensional spacetime. The choice of this space is given the 
José B. Almeida The hidden geometric character of relativistic quantum mechanics

character of first principle, justified solely by the consequences that can be derived from such choice and their consistency with experimental results. Given a metric space of any dimension, one can define monogenic functions, the natural extension of analytic functions to higher dimensions; such functions have null vector derivative and have previously been shown by other authors to play a decisive role in lower dimensional spaces.

All monogenic functions have null Laplacian by consequence; in an hyperbolic space this fact leads inevitably to a wave equation with plane-like solutions. This is also true for 5-dimensional spacetime and we will explore those solutions, establishing a parallel with the solutions of the free particle Dirac equation. For this purpose we will invoke the isomorphism between the complex algebra of $4 \times 4$ matrices, also known as Dirac's matrices. There is one problem with this isomorphism, because the solutions to Dirac's equation are usually known as spinors (column matrices) that don't belong to the $4 \times 4$ matrix algebra and as such are excluded from the isomorphism. We will show that a solution in terms of Dirac spinors is equivalent to a plane wave solution.

Just as one finds in the standard formulation, monogenic functions can be naturally split into positive/negative energy together with left/right ones. This split is provided by geometric projectors and we will show that there is a second set of projectors providing an alternate 4 -fold split. The possible implications of this alternate split are not yet fully understood and are presently the subject of profound research. 
José B. Almeida The hidden geometric character of relativistic quantum mechanics

\section{Introduction}

I have been advocating in recent papers that the majority of physics equations can be derived from an appropriately chosen geometry by exploration of the monogenic condition. Monogenic functions are not familiar to everybody but they are really the natural extension of analytic functions when one uses the formalism of geometric algebra [1, 2, 3, 4]; those functions zero the vector derivative defined on the algebra of the particular geometry under study.

In 5] I showed how special relativity and the Dirac equation could be derived from the monogenic condition applied in the geometric algebra of 5-dimensional spacetime $G_{4,1}$. An earlier paper [6] proved that the same condition in the same algebra was sufficient to produce a symmetry group isomorphic to the standard model gauge group; unfortunately this paper is incorrect in the formulation of particle dynamics but the flaw was recently corrected; [7] the same work introduces electrodynamics and electromagnetism in the monogenic formalism. Cosmological consequences were drawn from the addition of an hyperspherical symmetry hypothesis with the consequent choice of hyperspherical coordinates. 8] Summing up all those cited papers, I wrote a long book chapter; 9] the latest in the series is the derivation of energy states for the hydrogen atom from the monogenic condition. [10]

The present paper uses the 5D monogenic condition in 5D spacetime as a postulate and explores its consequences for quantum mechanics, establishing the conditions for equivalence to free particle Dirac's equation but going beyond that and opening paths that may lead to particle physics. 


\section{Some geometric algebra}

Geometric algebra is not usually taught in university courses and its presence in the

literature is scarce; good reference works are 1, 2, 3]. We will concentrate on the algebra of 5-dimensional spacetime because this will be our main working space; this algebra incorporates as subalgebras those of the usual 3-dimensional Euclidean space, Euclidean 4-space and Minkowski spacetime. We begin with the simpler 5-D flat space and progress to a 5-D spacetime of general curvature (see Appendix $\mathbb{C}$ for more details.)

The geometric algebra $G_{4,1}$ of the hyperbolic 5-dimensional space with signature $(-++++)$ is generated by the coordinate frame of orthonormal basis vectors $\sigma_{\alpha}$ such that

$$
\begin{aligned}
& \left(\sigma_{0}\right)^{2}=-1, \\
& \left(\sigma_{i}\right)^{2}=1, \\
& \sigma_{\alpha} \cdot \sigma_{\beta}=0, \quad \alpha \neq \beta .
\end{aligned}
$$

Note that the English characters $i, j, k$ range from 1 to 4 while the Greek characters $\alpha, \beta, \gamma$ range from 0 to 4 . See Appendix $₫$ for the complete notation convention used.

Any two basis vectors can be multiplied, producing the new entity called a bivector. This bivector is the geometric product or quite simply the product, and it is distributive. Similarly to the product of two basis vectors, the product of three different basis vectors produces a trivector and so forth up to the fivevector, because five is the spatial dimension.

We will simplify the notation for basis vector products using multiple indices, i.e. $\sigma_{\alpha} \sigma_{\beta} \equiv \sigma_{\alpha \beta}$. The algebra is 32-dimensional and is spanned by the basis

- 1 scalar, 1 , 
- 5 vectors, $\sigma_{\alpha}$,

- 10 bivectors (area), $\sigma_{\alpha \beta}$,

- 10 trivectors (volume), $\sigma_{\alpha \beta \gamma}$,

- 5 tetravectors (4-volume), i $\sigma_{\alpha}$,

- 1 pseudoscalar (5-volume), $\mathrm{i} \equiv \sigma_{01234}$.

Several elements of this basis square to unity:

$$
\left(\sigma_{i}\right)^{2}=\left(\sigma_{0 i}\right)^{2}=\left(\sigma_{0 i j}\right)^{2}=\left(\mathrm{i} \sigma_{0}\right)^{2}=1
$$

The remaining basis elements square to -1 :

$$
\left(\sigma_{0}\right)^{2}=\left(\sigma_{i j}\right)^{2}=\left(\sigma_{i j k}\right)^{2}=\left(\mathrm{i} \sigma_{i}\right)^{2}=\mathrm{i}^{2}=-1 .
$$

Note that the pseudoscalar i commutes with all the other basis elements while being a square root of -1 ; this makes it a very special element which can play the role of the scalar imaginary in complex algebra.

In 5-dimensional spacetime of general curvature, spanned by 5 coordinate frame vectors $g_{\alpha}$, the indices follow the conventions set forth in Appendix A] We will also assume this spacetime to be a metric space whose metric tensor is given by

$$
g_{\alpha \beta}=g_{\alpha} \cdot g_{\beta}
$$

the double index is used with $g$ to denote the inner product of frame vectors and not their geometric product. The space signature is still $(-++++)$, which amounts to saying that $g_{00}<0$ and $g_{i i}>0$. The coordinate frame vectors can be expressed as a linear combination of the orthonormed ones, for which reason we have

$$
g_{\alpha}=n^{\beta}{ }_{\alpha} \sigma_{\beta},
$$


where $n_{\alpha}^{\beta}$ is called the refractive index tensor or simply the refractive index; its 25 elements can vary from point to point as a function of the coordinates. 11] In this work we will not consider spaces of general curvature but only the connection-free ones, which can be designated as bent spaces; in those spaces we define the vector and covariant derivatives (see appendix (D).

\section{Electromagnetism as gauge theory}

The simplest example of a gauge theory is electromagnetism, so we will start by analysing this in the scope of monogenic functions. The monogenic condition in flat space is given by $[9]$

$$
\nabla \Psi=0
$$

which has plane wave like solutions of the type

$$
\Psi=\psi_{0} \mathrm{e}^{\mathrm{i} p_{\alpha} x^{\alpha}}
$$

where $p_{0}=E$ is interpreted as energy, $p_{4}=m$ as rest mass and $\mathbf{p}=\sigma^{m} p_{m}$ as 3-

dimensional momentum. Because the second order equation $\nabla^{2} \Psi=0$ must also be verified, we conclude easily that

$$
E^{2}-\mathbf{p}^{2}-m^{2}=0
$$

that is $E \sigma^{0}+p_{m} \sigma^{m}+m \sigma^{4}$ is a null vector.

A global symmetry of this equation is obtained with the transformation

$$
\Psi^{\prime} \mapsto \Psi \mathrm{e}^{\mathrm{i} \beta},
$$

where $\beta$ is a constant. It is easily verified that if $\Psi$ is monogenic so is $\Psi^{\prime}$ and the symmetry is global because $\beta$ is the same everywhere; the quantity $\exp (\mathrm{i} \beta)$ is a phase 
factor. If we allow $\beta$ to change with spacetime coordinates, $\beta=\beta\left(x^{\mu}\right)$, the monogenic condition will no longer be verified by $\Psi^{\prime}$

$$
\nabla \Psi^{\prime}=\nabla \Psi \mathrm{e}^{\mathrm{i} \beta}+\mathrm{i}^{\mu} \nabla \beta \Psi \mathrm{e}^{\mathrm{i} \beta} .
$$

We can define a local symmetry by changing the monogenic condition through replacement of the vector derivative by a covariant derivative which cancels out the extra term in Eq. (3.5); we do this as usual, by defining a vector field $A=\sigma^{\mu} A_{\mu}$ and writing the covariant derivative as

$$
\mathrm{D}={ }^{\mu} \nabla+\left(\sigma^{4}+\frac{q}{m} A\right) \partial_{4}
$$

where $A_{\mu}$ transforms as

$$
A^{\prime} \mapsto A-\frac{1}{q}{ }^{\mu} \nabla \beta
$$

In the two equations above $q$ and $m$ are charge and mass densities, respectively; in the non-dimensional units system $q=-1$ for an electron. Applying the extended monogenic condition to $\Psi^{\prime}$ we get

$$
\mathrm{D}^{\prime} \Psi^{\prime}=\nabla \Psi \mathrm{e}^{\mathrm{i} \beta}+\mathrm{i} q A \Psi \mathrm{e}^{\mathrm{i} \beta}=\mathrm{D} \Psi \mathrm{e}^{\mathrm{i} \beta} .
$$

So, obviously, if $\mathrm{D} \Psi$ is null, that is if $\Psi$ is monogenic in an extended sense, so is $\Psi^{\prime}$. Now, the covariant derivative in Eq. (3.6) can be associated with the reciprocal frame

$$
g^{\mu}=\sigma^{\mu}, \quad g^{4}=\sigma^{4}+\frac{q}{m} A_{\mu} \sigma^{\mu},
$$

which was introduced in Refs. 7, 9] to derive electrodynamics and electromagnetism from the monogenic condition. We see then that electromagnetic gauge invariance is a consequence of a non-orthonormed $g^{4}$ frame vector and can be accommodated in the refractive index formalism introduced above. 
José B. Almeida The hidden geometric character of relativistic quantum mechanics

\section{The Dirac equation}

In this section we examine the monogenic condition (3.1) and the solution (3.2) to establish the conditions under which they become equivalent to free particle Dirac's equation; the section ends with an indication of the procedure for the inclusion of an EM field. We will accept without explanation that the solution $\Psi$ has harmonic dependence on $x^{4}$ with a frequency equal to a particle's rest mass and write

$$
\Psi=\psi \mathrm{e}^{\mathrm{i} m x^{4}}
$$

At the present moment we cannot offer a plausible explanation for the existence of eigenvalues for the rest mass, corresponding to the various elementary particles; this is still a mystery with which we must live if we want to proceed. We note, however, that no existing theory offers a satisfactory explanation for the observed elementary particles' masses and so we are no worse than all other theories in that respect.

When the monogenic condition (3.1) is applied to $\Psi$ with the assumption above we get

$$
\left(\sigma^{0} \partial_{t}+\sigma^{m} \partial_{m}+\mathrm{i} \sigma^{4} m\right) \psi=0
$$

multiplying by $\mathrm{i} \sigma^{0}$ on the left

$$
\left(\mathrm{i} \partial_{t}+\mathrm{i} \sigma^{m 0} \partial_{m}-\sigma^{40} m\right) \psi=0 .
$$

Geometric algebra $G_{4,1}$ is isomorphic to the complex algebra of $4 * 4$ matrices [12], which means we can represent by matrix equations all the geometric equations in this paper. By resorting to matrix equations we will loose the geometric content inherent to geometric algebra; nevertheless such step is crucial if we want to understand the relationship with Dirac's equation because the latter uses matrix formalism. In order 
Table 1: Dirac-Pauli matrices.

$$
\begin{aligned}
& \alpha^{1}=\left(\begin{array}{llll}
0 & 0 & 0 & 1 \\
0 & 0 & 1 & 0 \\
0 & 1 & 0 & 0 \\
1 & 0 & 0 & 0
\end{array}\right), \quad \alpha^{2}=\left(\begin{array}{cccc}
0 & 0 & 0 & -\mathrm{i} \\
0 & 0 & \mathrm{i} & 0 \\
0 & -\mathrm{i} & 0 & 0 \\
\mathrm{i} & 0 & 0 & 0
\end{array}\right), \\
& \alpha^{3}=\left(\begin{array}{llll}
0 & 0 & 1 & 0 \\
0 & 0 & 0 & -1 \\
1 & 0 & 0 & 0 \\
0 & -1 & 0 & 0
\end{array}\right), \beta=\left(\begin{array}{cccc}
1 & 0 & 0 & 0 \\
0 & 1 & 0 & 0 \\
0 & 0 & -1 & 0 \\
0 & 0 & 0 & -1
\end{array}\right) .
\end{aligned}
$$

to make the transition be tween the two equations we need a matrix representation for geometric algebra elements; although there are many possible choices we will adopt the Dirac-Pauli representation, represented in Table 1. because it is commonly found in the literature. We can now associate geometric algebra elements to their matrix counterparts

$$
\sigma^{m 0} \equiv \alpha^{m}, \quad \sigma^{40} \equiv \beta .
$$

The different basis vectors of geometric algebra can then be given matrix equivalents 
Table 2: Matrix representation of basis vectors

$$
\begin{aligned}
\sigma^{0} \equiv & \left(\begin{array}{cccc}
0 & 0 & 1 & 0 \\
0 & 0 & 0 & 1 \\
-1 & 0 & 0 & 0 \\
0 & -1 & 0 & 0
\end{array}\right), \sigma^{1} \equiv\left(\begin{array}{cccc}
0 & 1 & 0 & 0 \\
1 & 0 & 0 & 0 \\
0 & 0 & 0 & -1 \\
0 & 0 & -1 & 0
\end{array}\right), \quad \sigma^{2} \equiv\left(\begin{array}{cccc}
0 & -\mathrm{i} & 0 & 0 \\
\mathrm{i} & 0 & 0 & 0 \\
0 & 0 & 0 & \mathrm{i} \\
0 & 0 & -\mathrm{i} & 0
\end{array}\right), \\
\sigma^{3} \equiv & \left(\begin{array}{cccc}
1 & 0 & 0 & 0 \\
0 & -1 & 0 & 0 \\
0 & 0 & -1 & 0 \\
0 & 0 & 0 & 1
\end{array}\right), \sigma^{4} \equiv\left(\begin{array}{cccc}
0 & 0 & -1 & 0 \\
0 & 0 & 0 & -1 \\
-1 & 0 & 0 & 0 \\
0 & -1 & 0 & 0
\end{array}\right) .
\end{aligned}
$$

by

$$
\begin{aligned}
\sigma^{0} & \equiv \mathrm{i} \alpha^{1} \alpha^{2} \alpha^{3} \beta, \\
\sigma^{1} & \equiv \mathrm{i} \alpha^{2} \alpha^{3} \beta, \\
\sigma^{2} & \equiv \mathrm{i} \alpha^{1} \alpha^{3} \beta, \\
\sigma^{3} & \equiv-\mathrm{i} \alpha^{1} \alpha^{2} \beta, \\
\sigma^{4} & \equiv \mathrm{i} \alpha^{1} \alpha^{2} \alpha^{3}
\end{aligned}
$$

resulting in the matrices of Table 2

The monogenic condition (3.1) can then be written in the alternative form

$$
\left(\mathrm{i} \partial_{t}+\mathrm{i} \alpha^{m} \partial_{m}-\beta m\right) \psi=0
$$

this can be read either as a geometric or matrix equation. In the latter case one recognizes that it has become Dirac's own equation in the absence of fields. There are 
problems however in associating the plane wave solutions given by Eq. (3.2) to those of Dirac's equation, mainly because in the geometric case the solutions will have $4 * 4$ matrix representations while the spinors associated with Dirac's solutions are column matrices which lay outside the matrix algebra. In order to bring Dirac's spinors into the matrix algebra we construct a matrix by placing the four solutions to Dirac's equation side by side

$$
\bar{\psi}=\left(\begin{array}{cccc}
\bar{\psi}_{1} & \bar{\psi}_{2} & \bar{\psi}_{3} & \bar{\psi}_{4}
\end{array}\right)
$$

where $\bar{\psi}_{i}$ are the four eigenvectors verifying the Dirac equation. The overbar is used, where necessary, to indicate Dirac equation elements that must be represented in their matrix form. Dirac's equation can now be written in its eigenvalue form

$$
\bar{A} \bar{\psi}=\bar{\psi} \bar{\Lambda},
$$

where $\bar{A}$ is the matrix representation of $\alpha^{m} p_{m}+\beta m$ and $\bar{\Lambda}$ is a diagonal matrix containing the eigenvalues of $\bar{A}$. This form of Dirac's equation is equivalent to four instances of the usual free particle equation, each with its own spinor solution; the non-zero elements in $\Lambda$ are obviously $\pm E$. From the equation above one concludes easily that matrix $\bar{A}$ can be decomposed as

$$
\bar{A}=\bar{\psi} \bar{\Lambda} \bar{\psi}^{-1} \text {. }
$$

We return now to Eq. (4.2) to verify what it implies for $\psi$. The first member contains the product of a null vector by some function, which must ultimately be null; this implies that $\psi$ must contain a null vector factor $E \sigma^{0}+p_{m} \sigma^{m}+m \sigma^{4}$, although other factors are not excluded. We then make the new assumption that $\psi$ is of the 
form

$$
\begin{aligned}
\psi & =\left(-E+p_{m} \sigma^{m 0}+m \sigma^{40}\right) \mathrm{e}^{\mathrm{i}\left(-E t+p_{m} x^{m}\right)} \\
& \equiv(-E \bar{I}+\bar{A}) \mathrm{e}^{\mathrm{i}\left(-E t+p_{m} x^{m}\right)} .
\end{aligned}
$$

Inserting into Eq. (4.6) we see that the following must be verified

$$
\bar{A}(E \bar{I}+\bar{A})=E(E \bar{I}+\bar{A})
$$

and so it must be

$$
\bar{A}^{2}=E^{2} \bar{I} .
$$

Replacing one of the $\bar{A}$ on the left hand side with Eq. (4.9)

$$
\bar{A} \bar{\psi} \bar{\Lambda} \bar{\psi}^{-1}=E^{2} \bar{I}
$$

Multiplying both sides on the right by $\bar{\psi} \bar{\Lambda}^{-1}$

$$
\bar{A} \bar{\psi}=E^{2} \bar{\psi} \bar{\Lambda}^{-1}
$$

But $E^{2} \bar{\Lambda}^{-1}=\bar{\Lambda}$ and so the monogenic equation is equivalent to the eigenvalue equation

$$
\bar{A} \bar{\psi}=\bar{\psi} \bar{\Lambda} .
$$

Remember that $\bar{\psi}$ is a matrix containing the four eigenvectors of $\bar{A}$ and $\bar{\Lambda}$ is diagonal with the eigenvalues of $\bar{A}$, so we have just written four instances of Dirac's equation in matrix form.

A geometric equivalent to Dirac's equation for a particle in an electromagnetic field can be immediately obtained by extension of the monogenic condition (3.1). If the derivative operator $\nabla$ is replaced by $\mathrm{D}$ from Eq. (3.6), the new geometric condition represents particles in fields, in the same way as Dirac's equation; this was proven 
José B. Almeida The hidden geometric character of relativistic quantum mechanics

for the case of the hydrogen atom in [10]. We say that we are applying an extended monogenic condition because the original monogenic condition is applied in a curved space with frame vectors given by Eq. (3.9).

\section{Energy and helicity splits}

When we imposed that $\bar{\psi}$ should have the four eigenvectors of $\bar{A}$ side by side we said nothing about ordering them because different ordering options only imply rearrangements of positive and negative elements in the diagonal matrix $\bar{\Lambda}$. We are then free to choose one of the possible orders that lead to

$$
\bar{\Lambda}=E \beta=E \sigma^{40} .
$$

It turns out that $\left(1+\sigma^{40}\right) / 2$ and $\left(1-\sigma^{40}\right) / 2$ are two orthogonal idempotents of $G_{4,1}$. This means that they are both elements that square to themselves, thus the idempotent designation, and their product is zero. In matrix representation, they are both diagonal matrices, the former with the top two elements equal to identity and the bottom pair equal to zero and the latter with the positions reversed. It is then clear that by multiplying Eq. 4.15) on the right by one of the idempotents we can select either the positive or negative energy equations from the set of four equations represented in the original form.

It is actually possible to define sets of four orthogonal idempotents in $G_{4,1}$ in different ways; one such possibility results from multiplying each of the energy split idempotents just defined by one of the pair $\left(1+\sigma^{3}\right) / 2$ and $\left(1-\sigma^{3}\right) / 2$. These idempotents select left or right spin solutions and we call this the helicity split. The set of four idempotents 
is then

$$
\begin{aligned}
& f_{1}=\frac{1}{4}\left(1-\sigma^{3}\right)\left(1-\sigma^{04}\right) ; \\
& f_{2}=\frac{1}{4}\left(1-\sigma^{3}\right)\left(1+\sigma^{04}\right) ; \\
& f_{3}=\frac{1}{4}\left(1+\sigma^{3}\right)\left(1+\sigma^{04}\right) ; \\
& f_{4}=\frac{1}{4}\left(1+\sigma^{3}\right)\left(1-\sigma^{04}\right) .
\end{aligned}
$$

Another set of four orthogonal idempotents is provided by the definitions

$$
\begin{aligned}
& e_{1}=\frac{1}{4}\left(1+\sigma^{012}\right)\left(1+\sigma^{034}\right) ; \\
& e_{2}=\frac{1}{4}\left(1+\sigma^{012}\right)\left(1-\sigma^{034}\right) ; \\
& e_{3}=\frac{1}{4}\left(1-\sigma^{012}\right)\left(1-\sigma^{034}\right) ; \\
& e_{4}=\frac{1}{4}\left(1-\sigma^{012}\right)\left(1+\sigma^{034}\right) .
\end{aligned}
$$

This set is not totally independent from the previous one, because the $\sigma^{034}$ element is present in both sets, but the two sets don't obviously overlap. In order to get some insight into the physical meaning of this second set we will have to look at some of the symmetries in $G_{4,1}$.

The isomorphism between geometric algebra $G_{4,1}$ and matrix algebra $M(4, C)$ allows us to state that unitary elements of the algebra are elements of symmetry group $U(4)$ and those with unit determinant are elements of symmetry group $S U(4)$. We don't need any further demonstration because we can always find a geometric algebra equivalent to any matrix element of $S U(4)$, however the result of this conversion is dependent on the particular assignment made between basis vectors and matrices and there are infinitely many ways of making such assignment. All elements of an $S U(4)$ group can be generated by a set of 15 generators; in matrix form, one frequent choice is shown in Table 3, 13] One verifies easily that there are 3 diagonal elements: $\lambda_{3}, \lambda_{8}$ 
and $\lambda_{15}$; since the four $f_{i}$ idempotents have diagonal matrix representations, they can be written as linear combinations of the 3 diagonal generators and unit, as follows

$$
\begin{aligned}
& f_{1}=\frac{1}{4}+\frac{\lambda_{3}}{2}+\frac{\lambda_{8}}{2 \sqrt{3}}+\frac{\lambda_{15}}{2 \sqrt{6}} ; \\
& f_{2}=\frac{1}{4}-\frac{\lambda_{3}}{2}+\frac{\lambda_{8}}{2 \sqrt{3}}+\frac{\lambda_{15}}{2 \sqrt{6}} ; \\
& f_{3}=\frac{1}{4}-\frac{\lambda_{8}}{\sqrt{3}}+\frac{\lambda_{15}}{2 \sqrt{6}} ; \\
& f_{4}=\frac{1}{4}-\sqrt{\frac{3}{2}} \lambda_{15} .
\end{aligned}
$$

The relations above would be equally true if we had chosen a different matrix assignment for basis vectors such that the idempotents were no longer represented by diagonal matrices. If we analyse the set of $e_{i}$ idempotents, it is clear that they are not represented by diagonal matrices but this is only circunstancial for we could have chosen a matrix assignment that would render $e_{i}$ diagonal instead of $f_{i}$. This is to conclude that any orthogonal set of 4 idempotents can be represented as linear combinations of privileged $S U(4)$ generators; reversing the relations above one gets

$$
\begin{aligned}
\lambda_{3} & =f_{1}-f_{2}, \\
\lambda_{8} & =\frac{f_{1}+f_{2}-2 f_{3}}{\sqrt{3}}, \\
\lambda_{15} & =\frac{f_{1}+f_{2}+f_{3}-3 f_{4}}{\sqrt{6}} .
\end{aligned}
$$

To end this subject we will just say that $e_{i}$ idempotents may encode isospin, strangeness and charm in a still unclear way. Greiner and Müller [13] encode these quantum numbers into the diagonal generators of $S U(4)$, which gives some substance to our claim. 


\section{Stable wavepackets}

The fact that particles appear with stable spatial distributions seems incompatible with a wavepacket description. It is widely known that even a Gaussian beam spreads out, in spite of remaining Gaussian; other distributions will not even conserve shape. Bohm and Hiley 14] suggested that a Gaussian distribution could be stabilized by influence of quantum vacuum but we will show next that the monogenic condition can produce naturally stable wavepackets without appealing to foreign mechanisms.

Following the reasoning in previous paragraphs, we expect a stationary particle to be described by a wavefunction of the type

$$
\Psi=\psi_{r}\left(x^{m}\right) \psi_{t}\left(x^{0}, x^{4}\right)
$$

where $\psi_{r}$ describes the spatial distribution and $\psi_{t}$ describes the propagation along $x^{4}$. Applying the monogenic condition we get

$$
\nabla \psi_{r} \psi_{t}+\vec{\nabla} \psi_{r} \overleftarrow{\psi}_{t}=0
$$

This equation is verified if each term on the left hand side is independently equal to zero. In the first term we note that $\nabla \psi_{r}=0$ defines 3-dimensional monogenic functions or spherical harmonics, fully described in Doran and Lasenby [1]. These functions have only scalar and bivector $\sigma^{12}$ terms and so we can argue that $\nabla$ in the second term commutes with $\psi_{r}$. This is true because the only derivatives to be considered are with respect to $x^{0}$ and $x^{4}$ and both $\sigma^{0}$ and $\sigma^{4}$ will commute with $\psi_{r}$. We are then led to solve $\nabla \psi_{t}=0$, which produces wave solutions propagating along $x^{4}$. We have then established several stable spatial distributions, propagating along

$x^{4}$, compatible with stationary particles. For non-stationary particles we can rotate the solutions in $4 \mathrm{D}$, maintaining stable spatial distributions. Although we don't have 
José B. Almeida The hidden geometric character of relativistic quantum mechanics

yet a clear interpretation of this possibility, we believe it is likely that it will help understand why particles exhibit stable spatial distributions.

\section{Conclusion and future work}

The use of 5-dimensional spacetime and monogenic functions as first principles allows the derivation of numerous equations of high physical significance; in this paper we explored some of those with relevance for quantum mechanics. The widely accepted principle of gauge invariance was shown to be fully equivalent to $5 \mathrm{D}$ space curvature, allowing the formulation of Maxwell's equations and electrodynamics in the framework of monogenic functions.

In respect to Dirac's equation our point of departure was the monogenic condition in 5D spacetime expressed by Eq. (3.1), which has plane wave type solutions. We then added the constraint that $x^{4}$ dependence should be harmonic, involving the particle's rest mass and we expressed that constraint by Eq. (4.1). The constant factor in plane wave solutions of the monogenic condition must include a null vector but it can include other factors; we added the constraint that there was also a $\sigma^{0}$ factor, resulting in the form given by Eq. (4.10). Those two constraints are enough to render the monogenic condition fully equivalent to free particle Dirac's equation in its conventional form; we can then say that the monogenic condition fully includes Dirac's equation but it allows more general solutions which may or may not have physical significance.

It was shown that any set of four orthogonal idempotents is closely related to the diagonal generators of $S U(4)$ in a particular matrix representation. A second set of four orthogonal idempotents was identified, independent of the energy/helicity set; it can be argued that this second set is related to the standard model gauge group of 
quarks bosons and hadrons. This is little more than a speculation at present and more work is needed in order to fully understand the meaning of the two superimposed symmetries. The paper ends with a demonstration that the monogenic condition produces non-expanding 4D wavepackets that may become very useful for modelling stable particles.

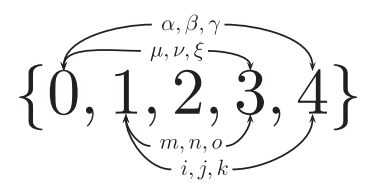

Figure 1: Indices in the range $\{0,4\}$ will be denoted with Greek letters $\alpha, \beta, \gamma$. Indices in the range $\{0,3\}$ will also receive Greek letters but chosen from $\mu, \nu, \xi$. For indices in the range $\{1,4\}$ we will use Latin letters $i, j, k$ and finally for indices in the range $\{1,3\}$ we will use also Latin letters chosen from $m, n, o$.

\section{A Indexing conventions}

In this section we establish the indexing conventions used in the paper. We deal with 5-dimensional space but we are also interested in two of its 4-dimensional subspaces and one 3-dimensional subspace; ideally our choice of indices should clearly identify their ranges in order to avoid the need to specify the latter in every equation. The diagram in Fig. 1 shows the index naming convention used in this paper; Einstein's summation convention will be adopted as well as the compact notation for partial derivatives $\partial_{\alpha}=\partial / \partial x^{\alpha}$. 


\section{B Non-dimensional units}

The interpretation of $t$ and $\tau$ as time coordinates implies the use of a scale parameter which is naturally chosen as the vacuum speed of light $c$. We don't need to include this constant in our equations because we can always recover time intervals, if needed, introducing the speed of light at a later stage. We can even go a step further and eliminate all units from our equations so that they become pure number equations; in this way we will avoid cumbersome constants whenever coordinates have to appear as arguments of exponentials or trigonometric functions. We note that, at least for the macroscopic world, physical units can all be reduced to four fundamental ones; we can, for instance, choose length, time, mass and electric charge as fundamental, as we could just as well have chosen others. Measurements are then made by comparison with standards; of course we need four standards, one for each fundamental unit. But now note that there are four fundamental constants: Planck constant $(\hbar)$, gravitational constant $(G)$, speed of light in vacuum $(c)$ and proton electric charge $(e)$, with which we can build four standards for the fundamental units.

Table 4 lists the standards of this units' system, frequently called Planck units, which the authors prefer to designate by non-dimensional units. In this system all the fundamental constants, $\hbar, G, c, e$, become unity, a particle's Compton frequency, defined by $\nu=m c^{2} / \hbar$, becomes equal to the particle's mass and the frequent term $G M /\left(c^{2} r\right)$ is simplified to $M / r$. We can, in fact, take all measures to be non-dimensional, since the standards are defined with recourse to universal constants; this will be our posture. Geometry and physics become relations between pure numbers, vectors, bivectors, etc. and the geometric concept of distance is needed only for graphical representation. 


\section{Some complements of geometric algebra}

In this section we expand the concepts given in Sec.2, introducing some useful relations and definitions. The geometric product of any two vectors $a=a^{\alpha} \sigma_{\alpha}$ and $b=b^{\beta} \sigma_{\beta}$ can be found making use of the distributive property and the already defined products of basis vectors

$$
a b=\left(-a^{0} b^{0}+\sum_{i} a^{i} b^{i}\right)+\sum_{\alpha \neq \beta} a^{\alpha} b^{\beta} \sigma_{\alpha \beta} .
$$

We notice it can be decomposed into a symmetric part, a scalar called the inner or interior product, and an anti-symmetric part, a bivector called the outer or exterior product.

$$
a b=a \cdot b+a \wedge b, \quad b a=a \cdot b-a \wedge b .
$$

Reversing the definition one can write inner and outer products as

$$
a \cdot b=\frac{1}{2}(a b+b a), \quad a \wedge b=\frac{1}{2}(a b-b a) .
$$

The inner product is the same as the usual "dot product," the only difference being in the negative sign of the $a_{0} b_{0}$ term; this is to be expected and is similar to what one finds in special relativity. The outer product represents an oriented area; in Euclidean 3 -space it can be linked to the "cross product" by the relation $\operatorname{cross}(\mathbf{a}, \mathbf{b})=-\sigma_{123} \mathbf{a} \wedge \mathbf{b}$; here we introduced bold characters for 3-dimensional vectors and avoided defining a symbol for the cross product because we will not use it again. We also used the convention that interior and exterior products take precedence over geometric product in an expression.

When a vector is operated with a multivector the inner product reduces the grade of each element by one unit and the outer product increases the grade by one. We will generalize the definition of inner and outer products below; under this generalized 
definition the inner product between a vector and a scalar produces a vector. Given a multivector $a$ we refer to its grade- $r$ part by writing $\langle a\rangle_{r}$; the scalar or grade zero part is simply designated as $\langle a\rangle$. By operating a vector with itself we obtain a scalar equal to the square of the vector's length

$$
a^{2}=a a=a \cdot a+a \wedge a=a \cdot a
$$

The definitions of inner and outer products can be extended to general multivectors

$$
\begin{aligned}
a \cdot b & =\sum_{\alpha, \beta}\left\langle\langle a\rangle_{\alpha}\langle b\rangle_{\beta}\right\rangle_{|\alpha-\beta|}, \\
a \wedge b & =\sum_{\alpha, \beta}\left\langle\langle a\rangle_{\alpha}\left\langle b>_{\beta}\right\rangle_{\alpha+\beta} .\right.
\end{aligned}
$$

Two other useful products are the scalar product, denoted as $\langle a b\rangle$ and commutator product, defined by

$$
a \times b=\frac{1}{2}(a b-b a) .
$$

In mixed product expressions we will use the convention that inner and outer products take precedence over geometric products as said above.

We will encounter exponentials with multivector exponents; two particular cases of exponentiation are specially important. If $u$ is such that $u^{2}=-1$ and $\theta$ is a scalar

$$
\begin{aligned}
\mathrm{e}^{u \theta}= & 1+u \theta-\frac{\theta^{2}}{2 !}-u \frac{\theta^{3}}{3 !}+\frac{\theta^{4}}{4 !}+\ldots \\
= & 1-\frac{\theta^{2}}{2 !}+\frac{\theta^{4}}{4 !}-\ldots\{=\cos \theta\} \\
& +u \theta-u \frac{\theta^{3}}{3 !}+\ldots\{=u \sin \theta\} \\
= & \cos \theta+u \sin \theta .
\end{aligned}
$$


Conversely if $h$ is such that $h^{2}=1$

$$
\begin{aligned}
\mathrm{e}^{h \theta}= & 1+h \theta+\frac{\theta^{2}}{2 !}+h \frac{\theta^{3}}{3 !}+\frac{\theta^{4}}{4 !}+\ldots \\
= & 1+\frac{\theta^{2}}{2 !}+\frac{\theta^{4}}{4 !}+\ldots\{=\cosh \theta\} \\
& +h \theta+h \frac{\theta^{3}}{3 !}+\ldots\{=h \sinh \theta\} \\
= & \cosh \theta+h \sinh \theta .
\end{aligned}
$$

The exponential of bivectors is useful for defining rotations; a rotation of vector $a$ by angle $\theta$ on the $\sigma_{12}$ plane is performed by

$$
a^{\prime}=\mathrm{e}^{\sigma_{21} \theta / 2} a \mathrm{e}^{\sigma_{12} \theta / 2}=\tilde{R} a R
$$

the tilde denotes reversion and reverses the order of all products. As a check we make $a=\sigma_{1}$

$$
\begin{aligned}
\mathrm{e}^{-\sigma_{12} \theta / 2} \sigma_{1} \mathrm{e}^{\sigma_{12} \theta / 2}= & \left(\cos \frac{\theta}{2}-\sigma_{12} \sin \frac{\theta}{2}\right) \sigma_{1} \\
& *\left(\cos \frac{\theta}{2}+\sigma_{12} \sin \frac{\theta}{2}\right) \\
& =\cos \theta \sigma_{1}+\sin \theta \sigma_{2} .
\end{aligned}
$$

Similarly, if we had made $a=\sigma_{2}$, the result would have been $-\sin \theta \sigma_{1}+\cos \theta \sigma_{2}$.

If we use $B$ to represent a bivector whose plane is normal to $\sigma_{0}$ and define its norm by $|B|=(B \tilde{B})^{1 / 2}$, a general rotation in 4-space is represented by the rotor

$$
R \equiv e^{-B / 2}=\cos \left(\frac{|B|}{2}\right)-\frac{B}{|B|} \sin \left(\frac{|B|}{2}\right) .
$$

The rotation angle is $|B|$ and the rotation plane is defined by $B$. A rotor is defined as a unitary multivector verifying $\tilde{R} R=1$; we are particularly interested in rotors with bivector components. It is more general to define a rotation by a plane (bivector) then by an axis (vector) because the latter only works in $3 \mathrm{D}$ while the former is applicable 
in any dimension. When the plane of bivector $B$ contains $\sigma_{0}$, a similar operation does not produce a rotation but produces a boost instead. Take for instance $B=\sigma_{01} \theta / 2$ and define the transformation operator $T=\exp (B)$; a transformation of the basis vector $\sigma_{0}$ produces

$$
\begin{aligned}
a^{\prime}= & \tilde{T} \sigma_{0} T=\mathrm{e}^{-\sigma_{01} \theta / 2} \sigma_{0} \mathrm{e}^{\sigma_{01} \theta / 2} \\
= & \left(\cosh \frac{\theta}{2}-\sigma_{01} \sinh \frac{\theta}{2}\right) \sigma_{0} \\
& *\left(\cosh \frac{\theta}{2}+\sigma_{01} \sinh \frac{\theta}{2}\right) \\
= & \cosh \theta \sigma_{0}+\sinh \theta \sigma_{1} .
\end{aligned}
$$

\section{Reciprocal frame and derivative operators}

A reciprocal frame is defined by the condition

$$
g^{\alpha} \cdot g_{\beta}=\delta^{\alpha}{ }_{\beta} .
$$

Defining $g^{\alpha \beta}$ as the inverse of $g_{\alpha \beta}$, the matrix product of the two must be the identity matrix, which we can state as

$$
g^{\alpha \gamma} g_{\beta \gamma}=\delta^{\alpha}{ }_{\beta} .
$$

Using the definition (2.4) we have

$$
\left(g^{\alpha \gamma} g_{\gamma}\right) \cdot g_{\beta}=\delta^{\alpha}{ }_{\beta}
$$

comparing with Eq. (D.1) we determine $g^{\alpha}$

$$
g^{\alpha}=g^{\alpha \gamma} g_{\gamma} .
$$

It would be easy to verify that it is also $g^{\alpha \beta}=g^{\alpha} \cdot g^{\beta}$ and $g_{\alpha}=g_{\alpha \gamma} g^{\gamma}$. 
In many situations of great interest the frame vectors $g_{\alpha}$ can be expressed in terms of an orthonormed frame given by Eqs. (2.1). If the frame vectors can be expressed as linear combination of the orthonormed ones we have

$$
g_{\alpha}=n^{\beta}{ }_{\alpha} \sigma_{\beta},
$$

where $n^{\beta}{ }_{\alpha}$ is called the refractive index tensor or simply the refractive index as said in the main text. When the refractive index is the identity we have $g_{\alpha}=\sigma_{\alpha}$ for the main or direct frame and $g^{0}=-\sigma_{0}, g^{i}=\sigma_{i}$ for the reciprocal frame, so that Eq. (D.1) is verified.

The first use we will make of the reciprocal frame is for the definition of two derivative operators. In flat space we define the vector derivative

$$
\nabla=\sigma^{\alpha} \partial_{\alpha}
$$

It will be convenient, sometimes, to use vector derivatives in subspaces of $5 \mathrm{D}$ space; these will be denoted by an upper index before the $\nabla$ and the particular index used determines the subspace to which the derivative applies; For instance ${ }^{m} \nabla=\sigma^{m} \partial_{m}=$ $\sigma^{1} \partial_{1}+\sigma^{2} \partial_{2}+\sigma^{3} \partial_{3}$. In 5-dimensional space it will be useful to split the vector derivative into its time and 4-dimensional parts

$$
\nabla=-\sigma_{0} \partial_{t}+\sigma^{i} \partial_{i}=-\sigma_{0} \partial_{t}+{ }^{i} \nabla
$$

Consistently with the boldface notation for 3 -dimensional vectors ${ }^{m} \nabla$ will be denoted by $\boldsymbol{\nabla}$. We will use over arrows, when necessary, to imply that the vector derivative is applied to a function which is not immediately on its right; for instance in $\vec{\nabla} A \overleftarrow{B}$ and in $\vec{B} A \overleftarrow{\nabla}$ the derivative operator is applied to function $B$.

The second derivative operator is called covariant derivative, sometimes also desig- 
José B. Almeida The hidden geometric character of relativistic quantum mechanics

nated by Dirac operator, and it is defined with recourse to the reciprocal frame $g^{\alpha}$

$$
\mathrm{D}=g^{\alpha} \partial_{\alpha}
$$

Taking into account the definition of the reciprocal frame (D.1) we see that the covariant derivative is also a vector. In cases where there is a refractive index, it will be possible to define both derivatives in the same space.

Vector derivatives can also be left or right multiplied with other vectors or multivectors. For instance, when $\nabla$ is multiplied by vector $a$ on the right the result comprises scalar and bivector terms $\nabla a=\nabla \cdot a+\nabla \wedge a$. The scalar part can be immediately associated with the divergence and the bivector part is called the exterior derivative; in the particular case of Euclidean 3-dimensional space it is possible to define the curl of a vector by $\operatorname{curl}(\mathbf{a})=-\sigma_{123} \boldsymbol{\nabla} \wedge \mathbf{a}$.

We define also second order differential operators, designated Laplacian and covariant Laplacian respectively, resulting from the inner product of one derivative operator by itself. The square of a vector is always a scalar and the vector derivative is no exception, so the Laplacian is a scalar operator, which consequently acts separately in each component of a multivector. For $4+1$ flat space it is

$$
\nabla^{2}=-\frac{\partial^{2}}{\partial t^{2}}+{ }^{i} \nabla^{2}
$$

In Minkowski spacetime it is usual to call D'Alembertian to $\nabla^{2}$ but we prefer to use the Laplacian designation for all spaces; this is consistent with the definition of tensor Laplacian found, for instance, in [15]. One sees immediately that a 4-dimensional wave equation is obtained zeroing the Laplacian of some function

$$
\nabla^{2} \Psi=\left(-\frac{\partial^{2}}{\partial t^{2}}+{ }^{i} \nabla^{2}\right) \Psi=0
$$


José B. Almeida The hidden geometric character of relativistic quantum mechanics

This procedure was used in Ref. [5] for the derivation of special relativity and extended in Ref. 7] to general curved spaces.

\section{References}

[1] C. Doran and A. Lasenby, Geometric Algebra for Physicists (Cambridge University Press, Cambridge, U.K., 2003).

[2] A. Lasenby and C. Doran, Physical applications of geometric algebra, handout collection from a Cambridge University lecture course, 2001, URL http://www.mrao.cam.ac.uk/ clifford/ptIIIcourse/index.html.

[3] D. Hestenes and G. Sobczyk, Clifford Algebras to Geometric Calculus. A Unified Language for Mathematics and Physics, Fundamental Theories of Physics (Reidel, Dordrecht, 1989).

[4] J. Ryan, Clifford analysis, in Lecture Notes on Clifford (Geometric) Algebras and Applications, edited by R. Ablamowicz and G. Sobczyk (Birkhäuser, Boston (USA), 2004), pp. 53-90.

[5] J. B. Almeida, Choice of the best geometry to explain physics, 2005, arXiviphysics/0510179

[6] J. B. Almeida, Geometric algebra and particle dynamics, in 7th International Conference on Clifford Algebras, ICCA7, edited by P. Anglès (To be published, Toulouse, France, 2005), arXiv math.GM/0504025.

[7] J. B. Almeida, Monogenic functions in 5-dimensional spacetime used as first prin- 
José B. Almeida The hidden geometric character of relativistic quantum mechanics

ciple: Gravitational dynamics, electromagnetism and quantum mechanics, 2006, arXiviphysics/0601078

[8] J. B. Almeida, Geometric drive of the universe's expansion, in 1st Crisis in Cosmology Conference, $C C C-I$, edited by E. Lerner and J. B. Almeida, Universidade do Minho (American Institute of Physics, Monção, Portugal, 2006), vol. 822 of AIP Conference Proceedings Series, pp. 110-122, arXiv physics/0507102

[9] J. B. Almeida, Can physics laws be derived from monogenic functions?, 2006, will appear in a book by Hadronic Press, arXivphysics/0601194

[10] J. B. Almeida, A geometric algebra approach to the hydrogen atom, 2006, arXivphysics/0602116.

[11] J. B. Almeida, The null subspace of $G(4,1)$ as source of the main physical theories, in Physical Interpretations of Relativity Theory - IX (London, 2004), arXivphysics/0410035.

[12] P. Lounesto, Clifford Algebras and Spinors, vol. 286 of London Mathematical Society Lecture Note Series (Cambridge University Press, Cambridge, U.K., 2001), 2nd ed.

[13] W. Greiner and B. Müller, Quantum Mechanics: Symmetries (Springer, Berlin, 2001), 2nd ed.

[14] D. Bohm and B. J. Hiley, The Undivided Universe (Routledge, London, U.K., 2005).

[15] G. B. Arfken and H. J. Weber, Mathematical Methods for Physicists (Academic Press, N. Y., 1995), 4th ed. 
Table 3: Generators for $S U(4)$ symmetry group

$$
\begin{aligned}
& \lambda_{1} \equiv\left(\begin{array}{cccc}
0 & 1 & 0 & 0 \\
1 & 0 & 0 & 0 \\
0 & 0 & 0 & 0 \\
0 & 0 & 0 & 0
\end{array}\right), \quad \lambda_{2} \equiv\left(\begin{array}{cccc}
0 & -\mathrm{i} & 0 & 0 \\
\mathrm{i} & 0 & 0 & 0 \\
0 & 0 & 0 & 0 \\
0 & 0 & 0 & 0
\end{array}\right), \quad \lambda_{3} \equiv\left(\begin{array}{cccc}
1 & 0 & 0 & 0 \\
0 & -1 & 0 & 0 \\
0 & 0 & 0 & 0 \\
0 & 0 & 0 & 0
\end{array}\right), \\
& \lambda_{4} \equiv\left(\begin{array}{cccc}
0 & 0 & 0 & 0 \\
0 & 0 & 1 & 0 \\
0 & 1 & 0 & 0 \\
0 & 0 & 0 & 0
\end{array}\right), \quad \lambda_{5} \equiv\left(\begin{array}{cccc}
0 & 0 & 0 & 0 \\
0 & 0 & -\mathrm{i} & 0 \\
0 & \mathrm{i} & 0 & 0 \\
0 & 0 & 0 & 0
\end{array}\right), \quad \lambda_{6} \equiv\left(\begin{array}{cccc}
0 & 0 & 1 & 0 \\
0 & 0 & 0 & 0 \\
1 & 0 & 0 & 0 \\
0 & 0 & 0 & 0
\end{array}\right) \\
& \lambda_{7} \equiv\left(\begin{array}{cccc}
0 & 0 & -\mathrm{i} & 0 \\
0 & 0 & 0 & 0 \\
0 & \mathrm{i} & 0 & 0 \\
0 & 0 & 0 & 0
\end{array}\right), \quad \lambda_{8} \equiv \frac{1}{\sqrt{3}}\left(\begin{array}{cccc}
1 & 0 & 0 & 0 \\
0 & 1 & 0 & 0 \\
0 & 0 & -2 & 0 \\
0 & 0 & 0 & 0
\end{array}\right), \quad \lambda_{9} \equiv\left(\begin{array}{cccc}
0 & 0 & 0 & 1 \\
0 & 0 & 0 & 0 \\
0 & 0 & 0 & 0 \\
1 & 0 & 0 & 0
\end{array}\right) \text {, } \\
& \lambda_{10} \equiv\left(\begin{array}{cccc}
0 & 0 & 0 & -\mathrm{i} \\
0 & 0 & 0 & 0 \\
0 & 0 & 0 & 0 \\
\mathrm{i} & 0 & 0 & 0
\end{array}\right), \lambda_{11} \equiv\left(\begin{array}{cccc}
0 & 0 & 0 & 0 \\
0 & 0 & 0 & 1 \\
0 & 0 & 0 & 0 \\
0 & 1 & 0 & 0
\end{array}\right), \quad \lambda_{12} \equiv\left(\begin{array}{cccc}
0 & 0 & 0 & 0 \\
0 & 0 & 0 & -\mathrm{i} \\
0 & 0 & 0 & 0 \\
0 & \mathrm{i} & 0 & 0
\end{array}\right) \text {, } \\
& \lambda_{13} \equiv\left(\begin{array}{cccc}
0 & 0 & 0 & 0 \\
0 & 0 & 0 & 0 \\
0 & 0 & 0 & 1 \\
0 & 0 & 1 & 0
\end{array}\right), \quad \lambda_{14} \equiv\left(\begin{array}{cccc}
0 & 0 & 0 & 0 \\
0 & 0 & 0 & 0 \\
0 & 0 & 0 & -i \\
0 & 0 & \mathrm{i} & 0
\end{array}\right), \quad \lambda_{15} \equiv \frac{1}{\sqrt{6}}\left(\begin{array}{cccc}
1 & 0 & 0 & 0 \\
0 & 1 & 0 & 0 \\
0 & 0 & 1 & 0 \\
0 & 0 & 0 & -3
\end{array}\right) \text {. }
\end{aligned}
$$


Table 4: Standards for non-dimensional units' system

\begin{tabular}{c|c|c|c} 
Length & Time & Mass & Charge \\
\hline$\sqrt{\frac{G \hbar}{c^{3}}}$ & $\sqrt{\frac{G \hbar}{c^{5}}}$ & $\sqrt{\frac{\hbar c}{G}}$ & $e$
\end{tabular}

